\title{
Spectral evolution of bright NS LMXBs
}

\author{
A. Paizis, ${ }^{a}$ R. Farinelli, ${ }^{b}$ L.I. Mainardi ${ }^{a}$ and L. Titarchuk ${ }^{b c}$ \\ ${ }^{a}$ INAF-IASF Milano, Italy \\ ${ }^{b}$ University of Ferrara, Italy \\ ${ }^{c} N A S A / G S F C$ Greenbelt, USA \\ E-mail:adaeiasf-milano.inaf.it
}

\begin{abstract}
Theoretical and observational support suggests that the spectral evolution of neutron-star LMXBs, including transient hard X-ray tails, may be explained by the interplay between thermal and bulk motion Comptonization. In this framework, we developed a new model for the X-ray spectral fitting XSPEC package which takes into account the effects of both thermal and dynamical (i.e. bulk) Comptonization, СомРТВ.

Using data from the INTEGRAL satellite, we tested our model on broad band spectra of a sample of persistently low magnetic field bright neutron star Low Mass X-ray Binaries, covering different spectral states. The case of the bright source GX 5-1 is presented here. Particular attention is given to the transient powerlaw-like hard X-ray (above $30 \mathrm{keV}$ ) tail that we interpret in the framework of the bulk motion Comptonization process, qualitatively describing the physical conditions of the environment in the innermost part of the system.
\end{abstract}

The Extreme sky: Sampling the Universe above $10 \mathrm{keV}$

October 13-17 2009

Otranto (Lecce) Italy 


\section{Introduction}

Low Mass X-ray Binaries (LMXB) hosting a Neutron Star, be they transient or persistent, show a variety of spectral states. A collection can be seen in Figure 1. The "intermediate state" (spectrum of GX 5-1) is particularly interesting because it merges two different spectral shapes: the typical thermal Comptonization bump, characterized by a $3-5 \mathrm{keV}$ electron plasma temperature and optical depth 10 , and a hard tail reaching much higher energies than expected from a $3 \mathrm{keV}$ Comptonizing plasma. Such hard-tails in the spectra of NS LMXBs (so-called Z sources) have been discovered by BeppoSAX ([2] and references therein) and subsequently confirmed and discovered in other sources with e.g. RXTE, INTEGRAL. Shape-wise, they have been fit with a power-law while physics-wise, several models have been proposed: e.g hybrid corona Comptonization [3], Synchrotron emission from jet electrons [4], Bulk motion Comptonization (e.g. [5], [6], [7]). In the latter model photons gain energy through first-order Fermi acceleration, the energization process producing the hard-tail occurs at the expense of the bulk kinetic energy of the in-falling matter and is not supplied by the gas internal energy. The strong point in favour of bulk Comptonization model is that it requires no

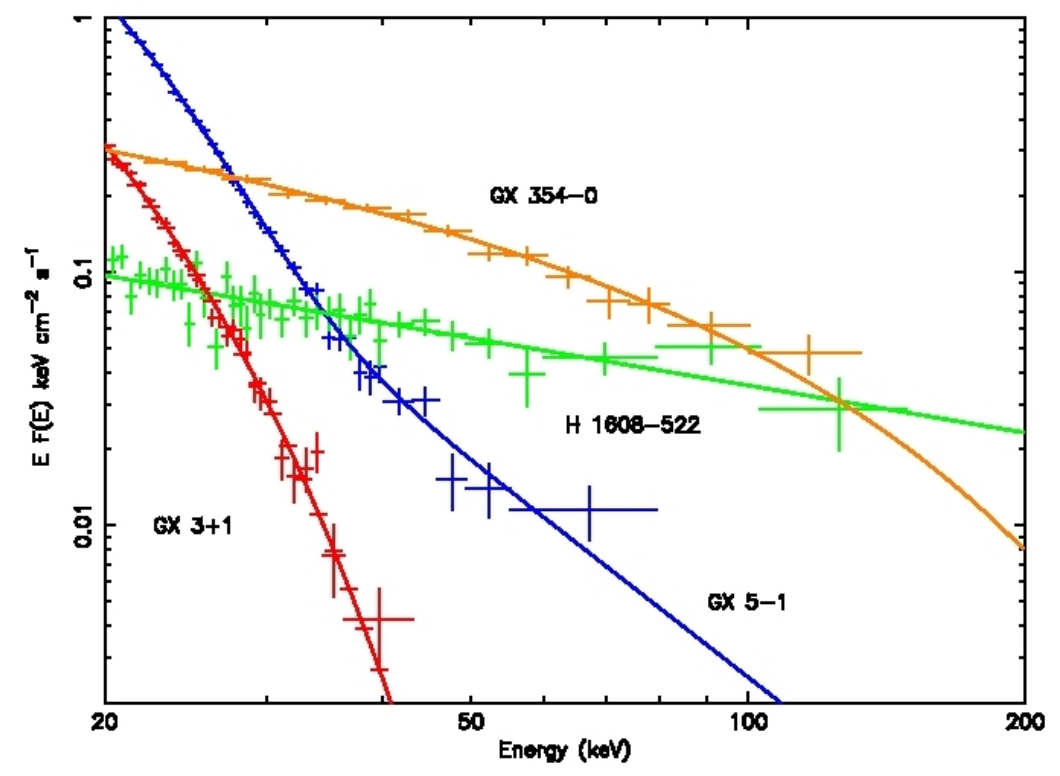

Figure 1: INTEGRAL IBIS/ISGRI average spectra of GX 354-0 ("pure" low/hard state, see text), H 1608522 (low/hard state), GX 5-1 (intermediate state) and GX 3+1 (very soft state) [1].

ad-hoc assumptions on the underlying physics (i.e. injection of non-thermal electron population by some unspecified mechanism) but in fact it can be explained in terms of first principles, using a full magneto-hydrodynamical treatment of the problem with appropriate boundary conditions, in particular by solving the radial momentum equation (Titarchuk \& Farinelli, in prep.). Moreover, this solution of the problem in a bounded configuration can also explain the temporal properties of NS LMXBs (e.g., [8]) which are difficult to explain with other models. Figure 2 shows an example of the velocity profile of the in-falling matter, with a clear increase closer to the NS surface where bulk motion Comptonization becomes more important. A complete set of computations for a grid of main physical parameters (accretion rate, etc) is in progress. 


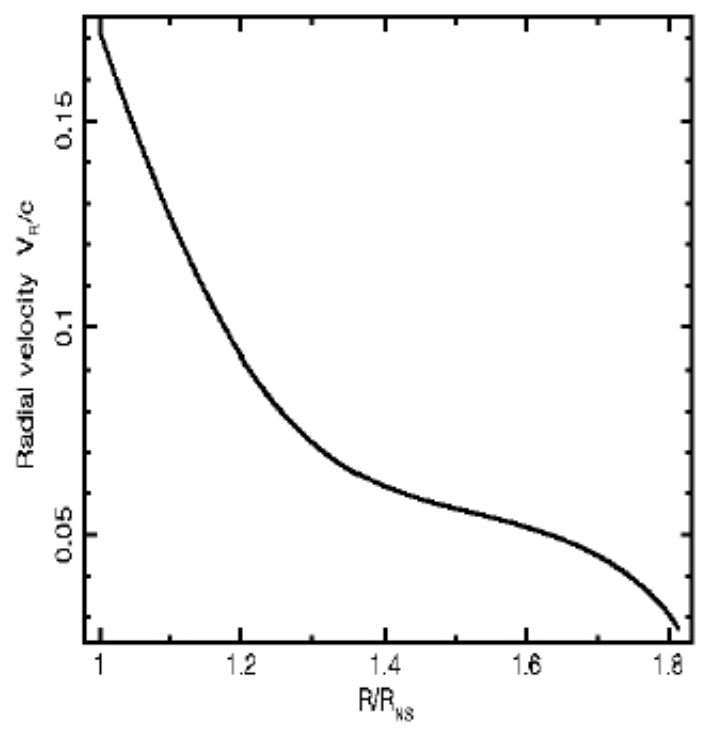

Figure 2: Radial velocity profile of the in-falling matter, obtained as a solution of the radial momentum equation for a given set of parameters which characterize the flow (Titarchuk \& Farinelli, in prep.)

\section{A unified physical scenario}

The first self-consistent physical scenario to explain the spectral evolution of persistent NS LMXBs, with particular attention to the transient hard-tails, has been proposed by [1]: the spectral evolution was explained as the result of the interplay of two components, thermal and bulk Comptonization, the latter, expected in the vicinity of the NS, being at the origin of the hard X-ray tail. The relative contribution of the two Comptonization regimes is proposed to be driven by the local accretion rate.

Thermal Comptonization is expected to originate in the external part of the corona (transition layer, TL) with seed photons coming mainly from the disk, while bulk Comptonization is produced in the inner part of the system, with seed photons from the NS and from the TL itself (see Figure 3). This scenario is consistent with results by [8] who used the characteristic (break and QPO) frequencies of the power density spectrum of 4 U 1728-34 to determine the geometric size of the configuration where the hard tail is formed.

\section{A new XSPEC Comptonization model: COMPTB}

We have developed a new XSPEC model that takes into account the effects of both thermal and bulk Comptonization [7] ${ }^{1}$. The model consists of the sum of direct BB emission and its Comptonization $\left(\mathrm{BB}^{*} \mathrm{G}\right)$ :

$$
F(E)=\frac{C_{n}}{A+1}(\underbrace{B B}_{(a)}+\underbrace{A \times B B * G}_{(b)})
$$

\footnotetext{
${ }^{1}$ COMPTB, http://heasarc.nasa.gov/docs/xanadu/xspec/models/comptb.html
} 


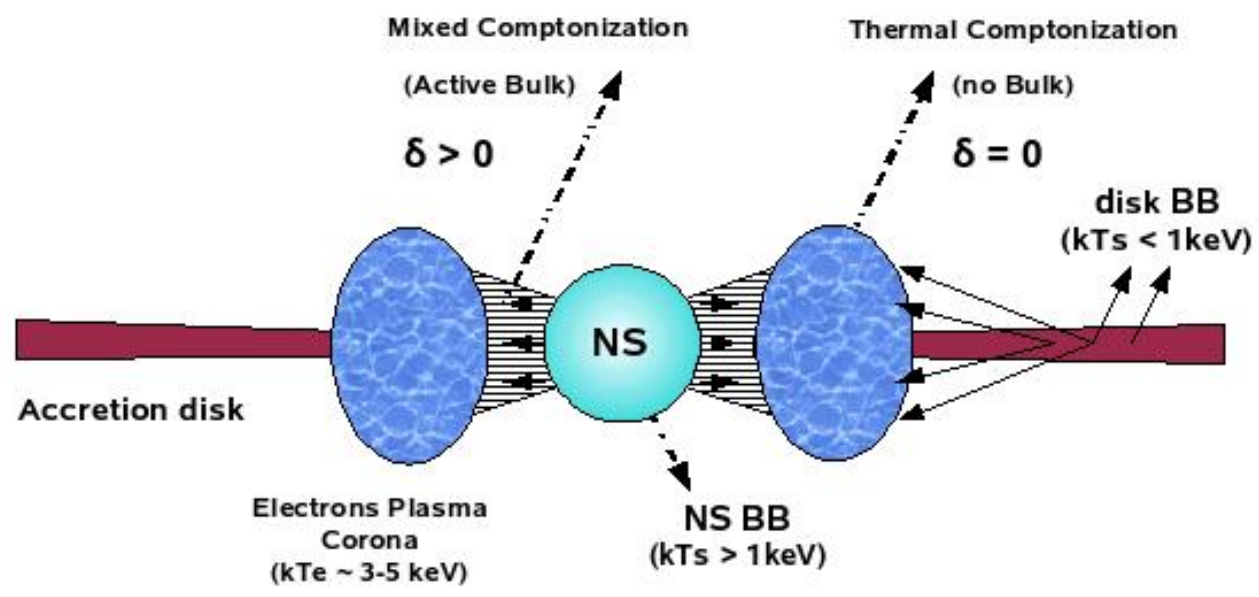

Figure 3: Schematic view of the proposed scenario for thermal and bulk Comptonization regions in LMXBs hosting NS [9].

where $C_{n}$ is the normalization constant, $A$ is the illumination factor, $C_{n} B B /(A+1)$ is the seed photon spectrum directly seen by the observer and not modified by Comptonization processes. $C_{n} A /(A+1) \times B B * G$ is the Comptonized spectrum obtained by convolution of a seed photon spectrum (blackbody, BB) with the Green's Function ${ }^{2}$, in order to evaluate the effect of thermal and bulk Comptonization on the seed photon field.

Parameters of the model are the seed photon temperature $k T_{s}$, the electron plasma temperature $k T_{e}$, the spectrum energy slope (i.e. overall Comptonization efficiency) $\alpha$ (photon index $\Gamma=\alpha+1$ ), the bulk parameter $\delta$ that quantifies the efficiency of bulk over thermal Comptonization and $\log A$, which assigns a different weight to the two components $(a)$ and $(b)$. This model enables the co-existence of the direct seed photon component and its Comptonized part, all obtained in a selfconsistent way. In Eq.[3.1] we note that for $\log A=-8$, we have only $(a)$, i.e. the direct seed photon component, while for $\log A=8$, the direct component is no longer visible and we have only thermal plus bulk Comptonization. In the case of $\delta=0$, the bulk Comptonization contribution is neglected, retaining only the thermal effects (equivalent to COMPTT, [10]).

\section{An application to the spectral evolution of NS LMXBs}

We have successfully applied the COMPTB model to several sources such as Sco X-1, GX 340+0, GX 354-0 [7], GX 5-1, GX 349+2, GX 13+1, GX 3+1, GX 9+1 [9], GS 1826-238 [11], Cyg X-2 [12], and GX 17+2 [13].

Figure 4 [9] shows the fit of the INTEGRAL spectra of GX 5-1 using two COMPTB components: the first one (dash) is thermal Comptonization of cold disk photons by the outer transition layer (see Fig. 3); the second one (dash-dot) describes the overall (thermal plus bulk) Comptonization of hotter seed photons close to the NS surface by the inner transition layer (delta $>0$, see

\footnotetext{
${ }^{2}$ The Green's Function, $G$, is the response of the system to the injection of a monochromatic line, see [7] for details.
} 

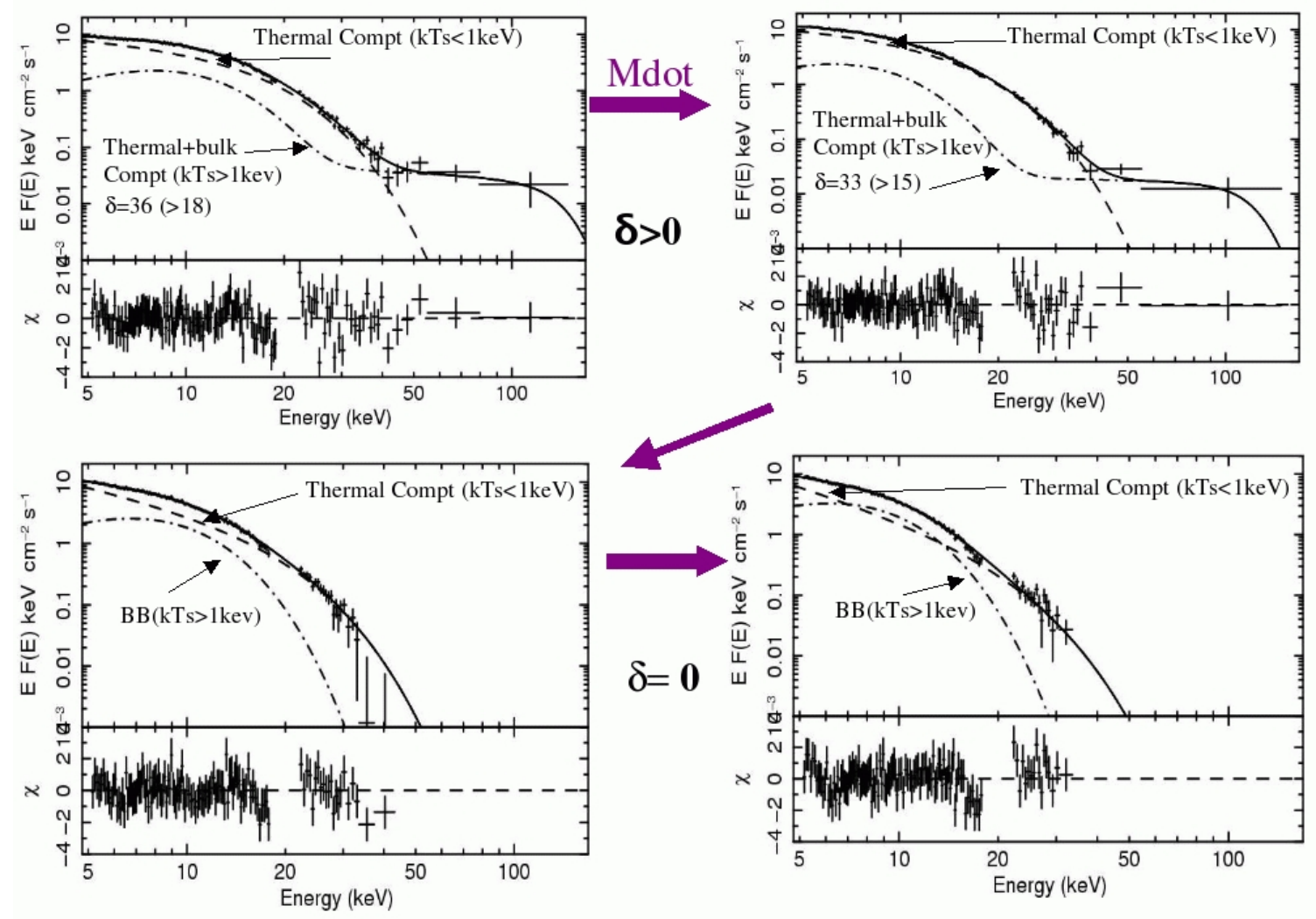

Figure 4: INTEGRAL spectra of GX 5-1 fit with two COMPTB models, one thermal, accounting for most of the energetic budget, and one bulk+thermal (accounting for the hard tail) or simple BB [adapted from 9].

Fig. 3). At increasing local accretion rates, the increasing radiation pressure from the NS inhibits the bulk inflow stopping the Comptonization, hence the hard tail component disappears and we see directly the NS emission (BB with kTs $>1 \mathrm{keV}$ ).

\section{Conclusions}

Our approach to explain in a self consistent way NS LMXBs is giving promising results. Understanding the origin of the hard tails in NS LMXBs is important to make a decisive step forward, from phenomenology (power-laws) to physics. This step forward will not make us understand hard tails alone, on the contrary: the hard-tails could be the observational feature that gives us the means to understand the mechanism of accretion flows in general. A large collaboration to investigate observational (e.g. [1], [7], [9], [11], [12], [13] and theoretical aspects (e.g. [5], [6], Titarchuk and Farinelli in prep.) of accretion flows in X-ray Binaries is in place.

Bulk Comptonization is gaining strong theoretical and observational support not only in the study of LMXB spectral evolution but also for High Mass X-ray Binaries (HMXB). A new theoretical model for accretion powered X-ray pulsars, based on bulk and thermal Comptonization occurring in the accreting shocked gas, was recently presented by [14]. The first application of this model on the spectrum of a HMXB (4U 0115+63) can be seen in [15]. 


\section{Acknowledgments}

AP acknowledges the Italian Space Agency financial support via contract I/008/07/0. This work has been partially supported by the Italian PRIN-INAF 2007 grant, "Bulk motion Comptonization models in X-ray Binaries: from phenomenology to physics", PI M. Cocchi.

\section{References}

[1] Paizis, A., Farinelli, R., Titarchuk, L., Courvoisier, T.J.-L., Bazzano, A., Beckmann, V., Frontera, F., Goldoni, P., Kuulkers, E., Mereghetti, S., Rodriguez, J., and Vilhu, O.: 2006, Astron. Astroph. 459, 187.

[2] Di Salvo, T. and Stella, L.: 2002, ArXiv Astrophysics e-prints, arXiv:astro-ph/0207219.

[3] Coppi, P.S.: 1999, High Energy Processes in Accreting Black Holes 161, 375.

[4] Markoff, S., Nowak, M.A., and Wilms, J.: 2005, Astrophys. J. 635, 1203.

[5] Titarchuk, L., Mastichiadis, A., and Kylafis, N.D.: 1996, Astronomy and Astrophysics Supplement Series 120, 171.

[6] Titarchuk, L., Mastichiadis, A., and Kylafis, N.D.: 1997, Astrophys. J. 487, 834.

[7] Farinelli, R., Titarchuk, L., Paizis, A., and Frontera, F.: 2008, Astrophys. J. 680, 602.

[8] Titarchuk, L. and Shaposhnikov, N.: 2005, Astrophys. J. 626, 298.

[9] Mainardi, L.I., Paizis, A., Farinelli, R., Kuulkers, E., Rodriguez, J., Hannikainen, D., Savolainen, P., Piraino, S., Bazzano, A., and Santangelo, A.: 2009, ArXiv e-prints, arXiv:0912.2707.

[10] Titarchuk, L.: 1994, Astrophys. J. 434, 570.

[11] Cocchi, M., Farinelli, R., Paizis, A., and Titarchuk, L.: 2009, ArXiv e-prints, arXiv:0911.0346.

[12] Farinelli, R., Paizis, A., Landi, R., and Titarchuk, L.: 2009, Astron. Astroph. 498, 509.

[13] Farinelli, R., Titarchuk, L., and Frontera, F.: 2007, Astrophys. J. 662, 1167.

[14] Becker, P.A. and Wolff, M.T.: 2007, Astrophys. J. 654, 435.

[15] Ferrigno, C., Becker, P.A., Segreto, A., Mineo, T., and Santangelo, A.: 2009, Astron. Astroph. 498, 825 . 